\title{
Ciudadanía comunicativa, espacio público mediático y realizadores locales: el caso del Plan de Fomento Audiovisual en Mendoza, Argentina
}

\author{
Rocío Belén Pérez Monteleone \\ FLACSO Ecuador
}

\section{Resumen}

Recibido: 8 de septiembre de 2015. Aceptado: 30 de diciembre de 2015.

El presente trabajo expone las principales conclusiones de la investigación de mi tesis de la Maestría en Comunicación de la Facultad Latinoamericana de Ciencias Sociales (FLACSO), sede Ecuador. El trabajo se titula "Instrumentos de políticas públicas de comunicación y realizadores audiovisuales locales argentinos: una contribución a la ciudadanía comunicativa”. Aquí se exponen parcialmente los principales hallazgos de esta investigación.

En el marco de las políticas públicas de comunicación recientemente sancionadas en Argentina (Ley de Servicios de Comunicación Audiovisual), la investigación propone un análisis de las potencialidades de los instrumentos resultantes de este proceso para ampliar las posibilidades de participación de instancias ciudadanas de producción audiovisual. Este objetivo se basa en la herramienta conceptual denominada "ciudadanía comunicativa", la cual profundiza la relación entre derecho, participación y espacio público mediático en un contexto atravesado por nuevas tecnologías y prácticas de comunicación globalizadas.

El caso de estudio analizado se basa en la implementación del Plan de Fomento y Promoción de Contenidos Audiovisuales Digitales, el cual tiene como objetivo financiar proyectos de realizadores independientes del interior del país. El interrogante que surge es la capacidad de este plan para contribuir al ejercicio de la ciudadanía comunicativa. Con este fin, se realizaron trece entrevistas a los ganadores del concurso ubicados en Mendoza, durante el período marzo-abril de 2014.

Palabras clave: ciudadanía comunicativa, espacio público mediático, realizadores locales, Ley 26.522, televisión digital, Argentina. 


\section{Communicative citizenship, public media space, and local professionals: the case of the Plan for Audiovisual Development in Mendoza, Argentina}

This paper shares the main conclusions from my thesis for my master's degree in Communication from the Ecuadorian branch of the Latin American Social Sciences Institute (FLACSO), which is titled, "Instruments of public communication policies and local audiovisual professionals in Argentina: a contribution to communicative citizenship." What follows are the main findings from my investigation.

Within the framework of public communication policies recently sanctioned in Argentina (the Audiovisual Communication Services Law), this investigation proposes an analysis of the potential for the instruments that have emerged from this process to broaden the possibilities for participation in audiovisual production by citizen groups. This investigative objective is based on the conceptual tool of "communicative citizenship," which deepens the relationship between rights, participation, and public media space in a context marked by new technologies and globalized communication practices.

The case study is based on the implementation of the Development and Promotion of Audiovisual Digital Content Law, which seeks to finance projects by independent professionals outside the country's capital. The question is whether this plan can really contribute to the exercise of communicative citizenship. To this end, thirteen interviews were conducted in March-April 2014 with the winners of the competition from Mendoza.

Keywords: communicative citizenship, public media space, local professionals, Law 26.522, digital television, Argentina.

\section{Cidadania comunicativa, espaço público midiático e realizadores locais: o caso do Plano de Fomento Audiovisual em Mendoza, Argentina}

Esse trabalho expõe as principais conclusões da pesquisa da minha tese da Maestria em Comunicação da Faculdade Latino-americana de Ciências Sociais (FLACSO), sede Equador. O trabalho se intitula "Instrumentos de políticas públicas de comunicação e realizadores audiovisuais locais argentinos: uma contribuição à cidadania comunicativa”. Aqui se expõem parcialmente os principais achados dessa pesquisa.

No marco das políticas públicas de comunicação recentemente sancionadas na Argentina (Lei de Serviços de Comunicação Audiovisual), a pesquisa propõe uma análise das potencialidades dos instrumentos resultantes desse processo para ampliar as possibilidades de participação de instancias cidadã de produção audiovisual. Esse objetivo está baseado na ferramenta conceptual denominada "cidadania comunicativa", a qual aprofunda a relação entre direito, participação e espaço público mediático num contexto atravessado por novas tecnologias e práticas de comunicação globalizadas.

O caso de estudo analisado se baseia na execução do Plano de Fomento e Promoção de Conteúdos Audiovisuais Digitais, o qual tem como objetivo financiar projetos de realizadores independentes do interior do país. O interrogante que surge é a capacidade deste 
plano para contribuir ao exercício da cidadania comunicativa. Com esse fim, se realizaram treze entrevistas aos ganhadores do concurso localizados em Mendoza, durante o período março-abril de 2014.

Palavras chave: cidadania comunicativa, espaço público mediático, realizadores locais, Lei 26.522, televisão digital, Argentina.

\section{Introducción ${ }^{1}$}

En las últimas décadas, los estados nacionales promueven políticas públicas en las que la comunicación se concibe como un derecho humano fundamental. A nivel latinoamericano, Uruguay, Bolivia, Ecuador, Argentina y Venezuela desarrollan legislaciones para regular el ámbito de la radiodifusión. En Argentina, este proceso se plasma en la sanción de dos instrumentos de políticas públicas: la Ley 26.522 de Servicios de Comunicación Audiovisual [LSCA] (Argentina. Ley 26.522, 2009) y la implementación de un modelo de televisión digital fuertemente impulsado por el Estado.

La LSCA busca revertir el escenario de concentración monopólica vigente; en el que la propiedad de los medios de comunicación se concentra en pocos grupos empresarios ${ }^{2}$. Se plantea además una ruptura con la producción audiovisual centralizada en Buenos Aires, buscando generar una apertura a la diversidad regional y la participación de actores excluidos de los medios comerciales. Con este fin, la ley reconoce tres tipos de prestadores: estatales, privados comerciales y privados no comerciales, reservando a este último sector el 33\% del espectro (artículos 21, 45 y 65).

En cuanto a la televisión digital, Argentina adopta la norma nipo-brasileña ISDBT mediante la firma de un convenio bilateral con Brasil, a través del cual recibe equipamientos y capacitación (Bizberge, Mastrini \& Becerra, 2011). Este sistema no solo implica la adopción de un estándar de transmisión sino que propone un conjunto de medidas destinadas a fortalecer la industria audiovisual y a ofrecer un servicio de televisión digital de libre acceso; mediante la creación Consejo Asesor Interministerial de Televisión, dependiente del Ministerio de Planificación ${ }^{3}$.

\footnotetext{
${ }^{1}$ El presente artículo expone las principales conclusiones de la investigación de mi tesis de la Maestría en Comunicación de la Facultad Latinoamericana de Ciencias Sociales (FLACSO), sede Ecuador. El trabajo se titula "Instrumentos de políticas públicas de comunicación y realizadores audiovisuales locales argentinos: una contribución a la ciudadanía comunicativa”. Aquí se exponen en parte los principales hallazgos de esta investigación. ${ }^{2}$ Para un detalle actualizado véase el último libro de Martín Becerra (2015).

3 A cargo del Consejo Asesor se genera un sistema gratuito de televisión digital abierta (TDA) mediante la instalación de 85 antenas digitales que cubren el $87 \%$ del territorio nacional, cuyo servicio puede recepcionarse a través de televisores de última generación (Télam,2014, sep. 22). La política de TDA contempla el acceso universal mediante la instalación de decodificadores (set top box) que permiten recibir la señal en equipos de televisión analógica. Estos se reparten desde 2009 entre la población más vulnerable (Sel, 2013).
} 
Los instrumentos de políticas públicas de comunicación se relacionan estrechamente con una nueva forma de comprender la participación ciudadana a partir del surgimiento de las sociedades red (Castells, 2005). A diferencia de lo que ocurría en las sociedades de posguerra, en las que el ejercicio del derecho se comprendía en relación con la participación electoral en un Estado-nación determinado (Marshall \& Bottone, 1998), los ciudadanos contemporáneos ejercen sus derechos a través de prácticas de consumo y comunicación globalizadas (Hopenhayn, 2000).

Esta nueva visión de la participación ciudadana es central en un debate académico en el que confluyen nociones provenientes del campo de las ciencias políticas y de la comunicación. En Argentina, esta reflexión dio origen a una intersección denominada "comunicación y ciudadanía" (Mata, 2006), en la que se pretende comprender el valor de las prácticas comunicativas actuales en las sociedades democráticas, atravesadas por las posibilidades de acceso a las nuevas tecnologías y a la alfabetización audiovisual (Aguirre \& Fernández, 2013).

El concepto clave para comprender los lineamientos generales de esta corriente es el de "ciudadanía comunicativa" o "ciudadanía comunicacional", el cual da cuenta de la importancia del acceso a bienes tecnológicos y a la producción de contenidos simbólicos en las sociedades modernas. El autor Gerardo Halpern define a este tipo de ciudadanía como "una herramienta de producción y de demanda de acceso e igualación de derechos, entendiendo por ello prácticas complejas, móviles y, producto de sus inherentes tensiones, en permanente redefinición" (2012, p. 230).

A través de la óptica de esta corriente intelectual, el acceso a los beneficios que proporcionan determinados instrumentos de políticas comunicacionales puede considerarse como un espacio donde se ejercen derechos fundamentales. Con este fin, se elaboró el diseño de un caso de estudio particular que permitiese dar cuenta de la relación que se genera entre los objetivos de una política pública de comunicación y las posibilidades de participación de la ciudadanía.

Un instrumento que resultó de especial interés fue el Plan de Fomento y Promoción de Contenidos Audiovisuales Digitales, el cual se creó en 2010 mediante la firma de un acuerdo entre el Consejo Asesor para la Televisión Digital Abierta, la Universidad Nacional de San Martín (UNSAM) y el Instituto Nacional de Cine y Artes Audiovisuales (INCAA). 
Esta herramienta es un concurso destinado a "desarrollar las capacidades profesionales de directores, productores y guionistas independientes y [...] fomentar la producción de contenidos para la televisión digital en todo el territorio nacional a fin de promover la diversidad cultural propia de sus diferentes regiones" (Instituto Nacional de Cine y Artes Audiovisuales [INCAA], 2011). El motor que impulsa el certamen es la federalización de los contenidos y los discursos televisivos, entendida como garantía de democratización de los recursos destinados al sector ${ }^{4}$.

El punto de partida de este trabajo es el cuestionamiento de la potencialidad del Plan de Fomento para modificar sustancialmente las posibilidades de participación de actores históricamente relegados de la comunicación comercial. El objetivo de este trabajo es responder al siguiente interrogante: ¿de qué manera los realizadores locales ejercen ciudadanía comunicativa en el marco del Plan de Fomento Audiovisual implementado en Argentina?

\section{Marco teórico}

Dentro de las múltiples entradas posibles para abordar el término ciudadanía, asumimos una visión republicana del concepto centrada en la participación ${ }^{5}$ $y$ en el acceso igualitario a los derechos mediante este enfoque:

La comunicación deja de ser un medio o un instrumento para el logro de fines políticos y pasa a ser condición esencial para la existencia misma de una comunidad política, para la definición de lo que se consideran bienes comunes, para la existencia de la democracia y de la ciudadanía misma (Segura, 2008, p. 5).

Segura afirma que "la noción de ciudadanía viene asociada al concepto de espacio público en sus dimensiones urbana y mediática” (2008, p. 6). Este punto es de particular relevancia, ya que la participación ciudadana no se daría solo a través de los canales clásicos de representación; por el contrario, implicaría la participación activa en un "espacio público mediático"6 en el que los ciudadanos

\footnotetext{
${ }^{4}$ Con este fin, el concurso divide al país en seis regiones: Centro metropolitano, Centro norte, Noreste, Noroeste, Nuevo Cuyo y Patagonia. A lo largo de las cuatro emisiones anuales concretadas hasta la fecha se preseleccionan y se subsidian proyectos dentro de cada bloque regional en las siguientes convocatorias: series de documentales temáticos, series de ficciones federales, serie de documental federal temático, ciclos de programas infantiles y unitarios documentales. Esta modalidad concretó cerca de 2000 horas de contenidos (Sel, 2013, p. 4).

${ }^{5}$ Debido a cuestiones de extensión, este trabajo no abordará en profundidad la noción de participación.

${ }^{6}$ A falta de un concepto más pertinente, tendremos en cuenta esta categoría al proceso de cambio y convergencia que experimenta la industria audiovisual a partir de la digitalización de los contenidos y los avances tecnológicos. Plataformas como internet, la televisión digital y los dispositivos móviles (smartphone, ordenador, tablet) hacen necesaria la creación de contenidos que puedan visualizarse en todas las pantallas. A su vez, estos cambios posibilitan una mayor participación e interacción de las audiencias como generadoras de contenidos (Barceló \& Sánchez, 2010, p. 181).
} 
ejercen el derecho a utilizar y acceder a cualquier medio o canal para expresar sus ideas y opiniones, a manejar "lenguajes mediales", a participar en la elaboración de las políticas de comunicación y a contar con condiciones tecnológicas, jurídicas y económicas que garanticen este derecho (Camacho, 2007, p. 37).

En este sentido, consideramos que la posibilidad de producir bienes culturales mediante la creación de productos audiovisuales es una forma prioritaria de ejercicio de la ciudadanía comunicativa. La toma de decisiones en el ámbito de la producción audiovisual implica: a) la ocupación activa de un espacio público, durante años reservado a otro tipo de intereses; b) la apropiación de tecnologías y saberes varios con un impacto decisivo en la democratización de estos instrumentos; c) la construcción de nuevos relatos y representaciones de los intereses, reclamos y realidades colectivas; d) el rol protagónico del Estado, quien debe garantizar condiciones de acceso igualitario.

En este análisis se tienen en cuenta especialmente las posibilidades de participación en el espacio público mediático de grupos sociales subalternos; a los que Calderón, Hopenhayn \& Ottone (1996, p. 96) denominan “instancias de producción ciudadanas". Estas instancias deben ser comprendidas en un sentido amplio y su principal característica es la de plasmar en sus relatos audiovisuales una lógica diferente a la mercantil, en la búsqueda de contribuir a la democratización de las sociedades.

Dentro de esta categoría podemos encontrar a sujetos variados que devienen en productores audiovisuales a partir de sus necesidades específicas de comunicación. Entre ellos podemos mencionar a actores colectivos como los movimientos sociales, los pueblos originarios, las instituciones educativas, las asociaciones civiles y las instancias de producción comunitaria de toda clase.

\section{Metodología}

En primer lugar, fue central para esta investigación establecer un criterio de selección geográfico que permitiera comprender el impacto del plan de fomento. La dicotomía Buenos Aires versus El interior fue resuelta a través del análisis de los principales polos de producción audiovisual del país y cómo se distribuyen en ellos los ganadores de los concursos. Luego de esta indagación se llegó a la conclusión de que Mendoza, capital productora de la región de Cuyo, concentra el más alto número de ganadores a nivel nacional: en la edición 2010, once proyectos fueron premiados en la provincia en varias categorías de los trece totales que se le 
asignaron a la región (Escribiendocine, 2010, dic. 4). Esta hegemonía se mantuvo a lo largo de las emisiones posteriores.

Frente a esta concentración, surgen algunos interrogantes que buscamos develar en el proceso investigativo, los cuales deben entenderse como "criterios" (Guber, 2004) de carácter cualitativo y flexible. Estos son:

¿Quiénes son los ganadores de estos concursos? ¿Cuál es su procedencia, formación educativa y experiencia laboral? ¿Cuál es su trayectoria como beneficiarios de políticas de fomento al audiovisual? Estos actores ¿participan activamente en el espacio público mediático? ¿Qué visión tienen los realizadores de la participación ciudadana en la producción audiovisual? Finalmente ¿cuál es su relación con instancias de producción ciudadana?

Como unidad de estudio (Guber, 2004, p. 107) nos centramos en los realizadores audiovisuales localizados en la capital de la provincia. La muestra seleccionada estuvo compuesta por trece ganadores de las emisiones 2010 y 2011 del Plan de Fomento, en los rubros de series de ficción y series de documentales federales.

La técnica empleada consistió en la realización de entrevistas cualitativas semiestructuradas, las cuales se llevaron a cabo mediante la interacción directa con los actores involucrados (Bressers \& Toole, 2007, p. 150) en el período de marzo-abril de 2014. Se decidió mantener el anonimato de los realizadores para evitar posibles enfrentamientos o rispideces con el Instituto Nacional de Cine y Artes Audiovisuales, gestor de los concursos.

\section{Resultados de investigación}

\section{Características generales}

\section{a) Procedencia}

En primer lugar examinaremos la procedencia de los ganadores ${ }^{7}$ de acuerdo con tres criterios: Mendoza, Buenos Aires y otras provincias. Los resultados se plasman en el gráfico 1, en el que se puede observar que la gran mayoría de los entrevistados $(76,9 \%)$ son originarios de la provincia. En cuanto a los porteños radicados, representan el 15,4\% del total y los provincianos radicados, el 7,7\%.

\footnotetext{
${ }_{7}$ Como garantía de federalismo, los realizadores deberán tener su domicilio legal constituido en la región de Cuyo para acceder al concurso (artículo 9, Bases del concurso "Series de documentales federales", 2011).
} 


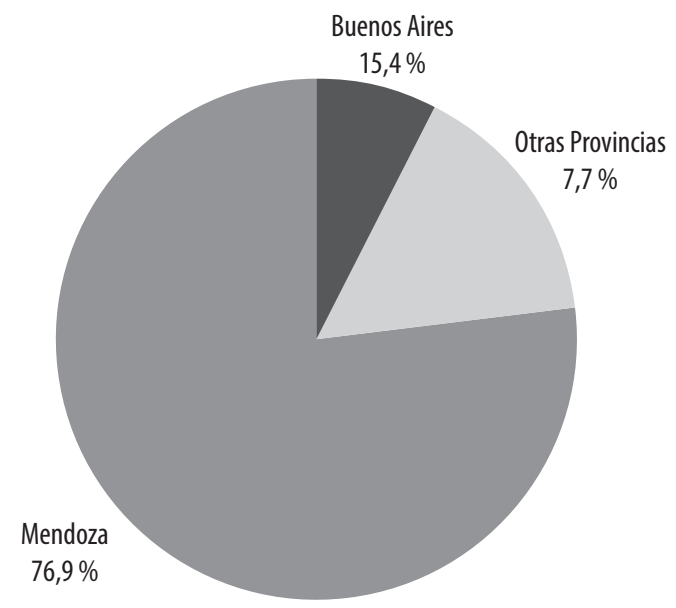

Gráfico 1. Realizadores según procedencia.

\section{b) Formación educativa}

Este punto también es de interés ya que permite comprender cuál es el nivel de especialización profesional necesario para acceder a los concursos. En este sentido, todos los entrevistados pasaron por una instancia de formación superior, en la gran mayoría de los casos completada (76,9\%) según se verifica en el gráfico 2.

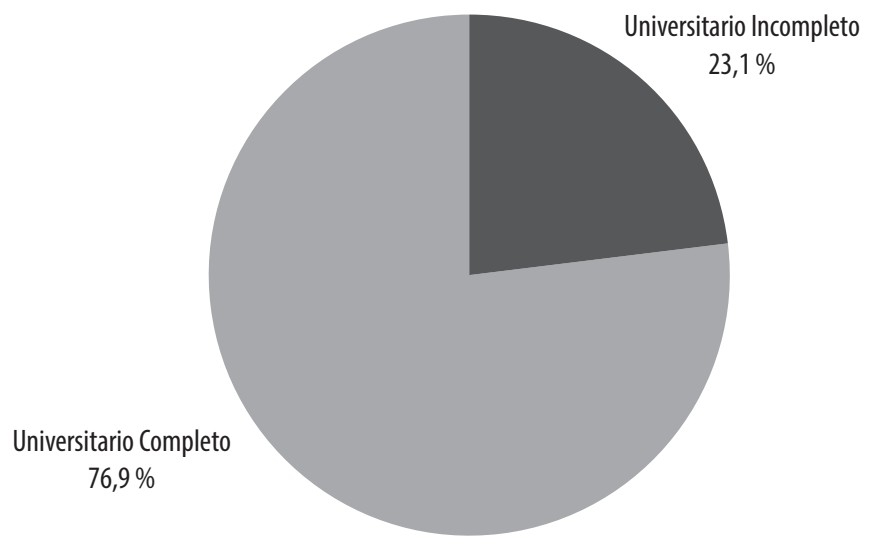

Gráfico 2. Realizadores según formación educativa. 
En cuanto a la procedencia de las instituciones educativas, un porcentaje importante de los entrevistados $(30,8 \%)$ tuvo la posibilidad de abandonar la provincia para realizar sus estudios superiores en Buenos Aires, estableciendo vínculos profesionales y personales en la capital. Sin embargo, el porcentaje de aquellos que permanecieron en la provincia y accedieron a instancias de formación local es alto, ya que corresponde al $69,2 \%$ (gráf. 3).

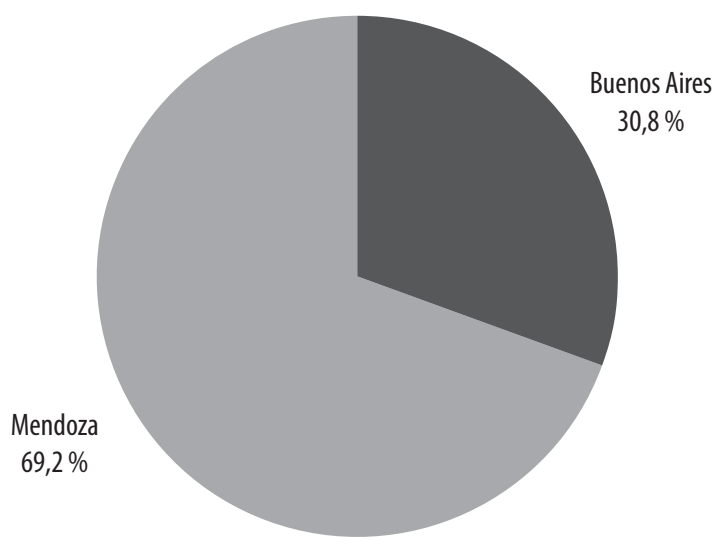

Gráfico 3. Procedencia de las instituciones educativas.

\section{c) Experiencia laboral}

Otra variable importante a analizar está constituida por el lugar de experiencia laboral de los realizadores, es decir, el ámbito en el que se formaron profesionalmente. En el 23,1\% de los casos encontramos a trabajadores mendocinos radicados en Buenos Aires por períodos de tiempo de entre 5 y 15 años. En muchos casos se trata de aquellos estudiantes provincianos que se fueron a estudiar a la capital, tanto de Mendoza como de otras ciudades del interior (7,7\%). El grueso de la torta está ocupado por el $53,8 \%$ correspondiente a los trabajadores locales que no abandonaron su lugar de origen, pero que mantuvieron fluidas relaciones con proyectos situados en Buenos Aires. El 15,4\% restante corresponde a trabajadores bonaerenses radicados en Mendoza en los últimos años, tanto por motivos personales como profesionales (gráf. 4). 


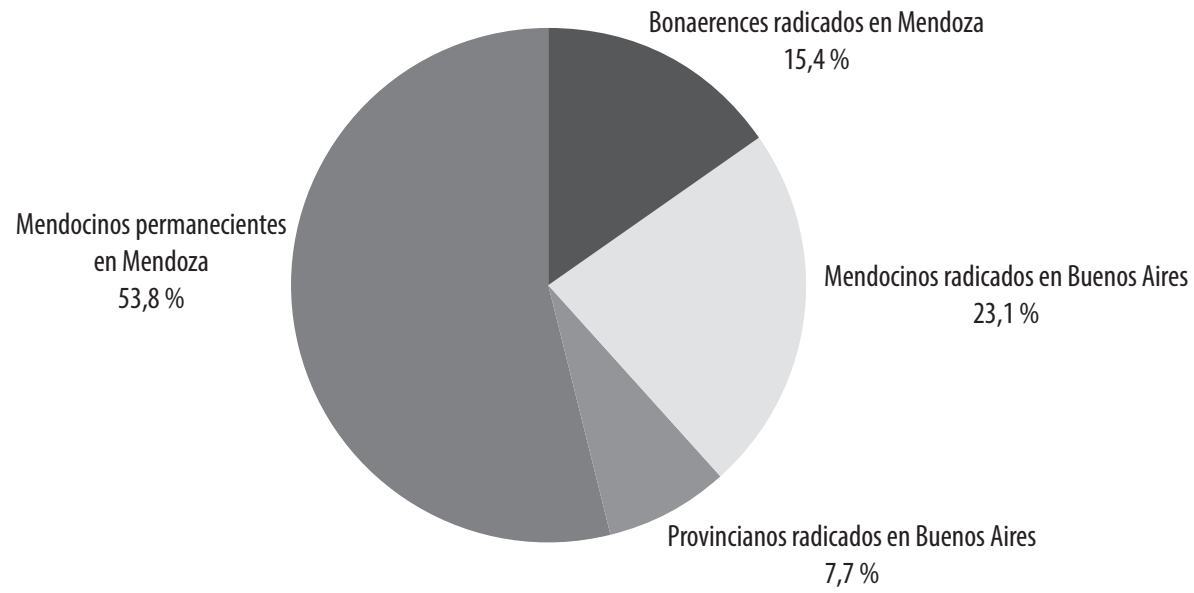

Gráfico 4. Lugar de experiencia laboral.

\section{d) Trayectoria previa en proyectos financiados por el Estado}

Es importante destacar que el 69,2\% de los entrevistados había participado alguna vez de políticas de fomento, mientras que el 30,8\% restante se presenta por primera vez a un concurso público (gráf. 5).

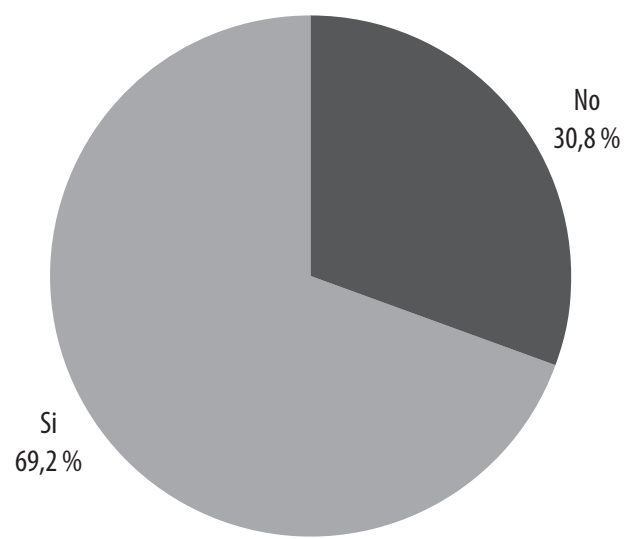

Gráfico 5. Participación en proyectos previos financiados por el Estado. 


\section{Participación}

Puede considerarse que el $100 \%$ de los entrevistados participa en el espacio público mediático, ya que sus trabajos obtienen en menor o mayor grado algún tipo de visibilidad. Los medios a través de los cuales circulan los productos una vez terminados incluyen canales de aire analógicos y digitales, públicos y privados (locales y nacionales), televisoras extranjeras e internet (a través del $\mathrm{BACUA}^{8} \mathrm{y}$ el portal de Contenidos Audiovisuales Digitales $[\mathrm{CDA}]^{9}$ ). Otro dato relevante es la visión que tienen los realizadores audiovisuales de la producción audiovisual como espacio de participación de la ciudadanía (gráf. 6). Existen tres criterios en los que pudimos englobar las respuestas:

a. Un espacio de participación activa de los actores sociales a través de instancias de capacitación audiovisual $(15,4 \%)$.

b. Un espacio de comercio, en el que la ciudadanía puede beneficiarse mediante la prestación de servicios como alquiler de locaciones, transporte o catering $(7,7 \%)$.

c. Un espacio de oferta de contenidos de calidad para espectadores que circulan a través de los medios masivos (69,2\%).

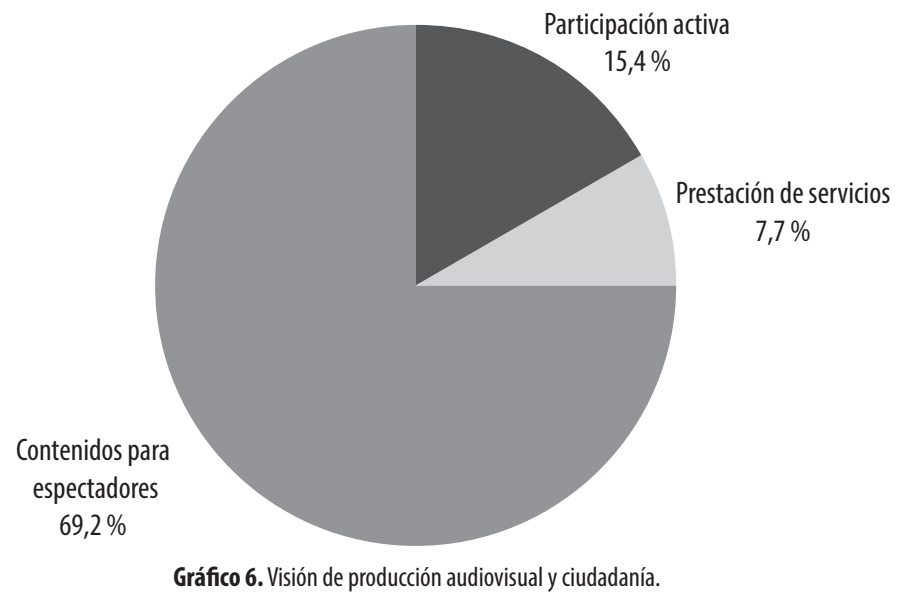

\footnotetext{
8 Es un reservorio digital de libre acceso y distribución gratuita que funciona a través de la sesión de derecho de los autores.

9 Es un sitio web que permite la visualización y descarga de contenidos financiados por el Estado argentino de manera gratuita bajo la modalidad "video on demand".
} 
La relación de los actores con instancias de producción ciudadana aparece como una variable en la que podemos desglosar 3 categorías (gráf. 7):

a. Activa: aquí incluimos a los realizadores que han participado en instancias ciudadanas a través de la militancia, independientemente de su labor audiovisual $(38,5 \%)$.

b. A través de la realización $(30,8 \%)$ : se vinculan con instancias de producción ciudadana incorporándolas como protagonistas en sus relatos.

c. Sin relación $(30,8 \%)$.

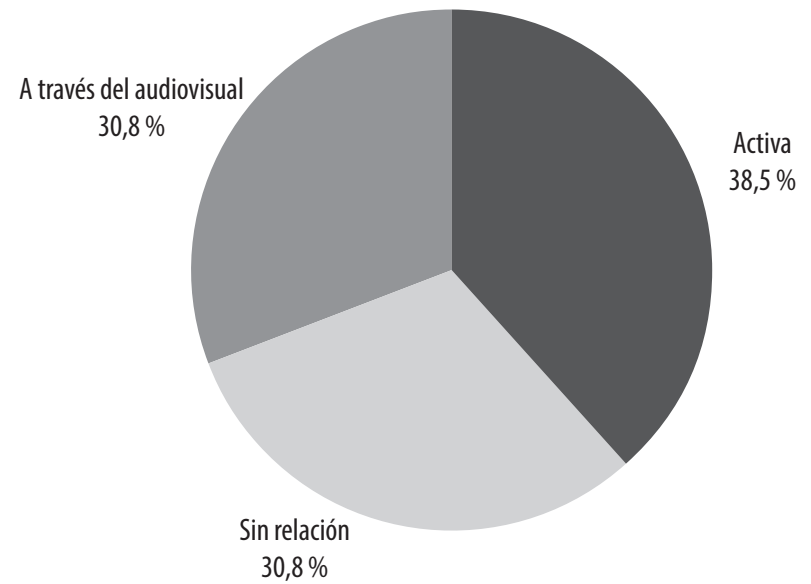

Gráfico 7. Participación en instancias ciudadanas.

\section{Lectura de los datos, hallazgos y conclusiones}

A partir de todo lo expuesto, se evidencia que el Plan de Fomento es una política en la que la producción de bienes audiovisuales es comprendida como un sector industrial. Este rubro es subsidiado para obtener resultados de alta calidad con el fin de garantizar las ventas de series argentinas a nivel regional y global en formato full HD.

Desde esta óptica, el audiovisual no sería un espacio de participación ciudadana; por el contrario: la ciudadanía es concebida como un espectador pasivo, cuyo ejercicio del derecho estaría supeditado a recibir contenidos de calidad a través de 
los medios masivos. Este punto se evidencia en la visión que tienen el $69,2 \%$ de los realizadores de la participación de instancias de producción ciudadana. De esta manera, se busca ofrecer al ciudadano-consumidor otras alternativas televisivas, que se suponen estarían ligadas a sus intereses de representación colectiva.

Con este fin, la política audiovisual otorga un monto considerable de dinero para la realización de proyectos en el interior del país; fomentando el surgimiento de una élite relativamente homogénea de realizadores ligados al sector comercial, con ciertas características comunes: alto nivel educativo (en el 76,9\% de los casos son profesionales universitarios), trayectoria previa en instancias de financiación $(69,2 \%)$ y amplia experiencia laboral tanto en Mendoza como en Buenos Aires. Este grupo ilustrado funcionaría como un intermediario entre la representación de los relatos y narrativas regionales en la que pueden o no intervenir instancias de producción ciudadanas retratadas como personajes.

La principal consecuencia de este enfoque es que la democratización se concibe como "federalización", la cual se resume en la distribución por regiones del presupuesto audiovisual nacional. En este sentido, el concurso es positivo, ya que un alto porcentaje de los entrevistados es oriundo de Mendoza (76,9\%); se formó en instituciones de educación superior provinciales $(69,2 \%)$ y se desarrolló profesionalmente en la región (53,8\%). A pesar de la presencia de realizadores provenientes de Buenos Aires y formados en la capital argentina, podemos afirmar que el Plan de Fomento tiene un impacto decisivo en el desarrollo incipiente de la industria local a nivel técnico, económico y humano.

Sin embargo, el certamen no tiene ningún impacto en la incorporación de grupos con menores capacidades y recursos. Como aporte, podemos plantear sugerencias prácticas para desarrollar un plan en el que se amplíe el ejercicio de la ciudadanía comunicativa:

a) De acuerdo con el artículo 124 de la LSCA, se conforma un Consejo Consultivo Honorario de los medios públicos, el cual comenzó a operar el 15 de enero a través del Decreto 51/15. El consejo está conformado por personalidades de la comunicación social y audiovisual universitaria; sindicatos de trabajadores del rubro, organismos representativos de las audiencias, organizaciones de derechos humanos y pueblos originarios, y un delegado correspondiente a cada una de las regiones del país (Señales, 2015, ene. 21). A nuestro parecer, esta instancia sería un ámbito privilegiado para debatir propuestas que enriquezcan la política audiovisual desde un punto de vista ciudadano. 
b) Como parte del articulado de los concursos es imprescindible iniciar la búsqueda de mecanismos que integren a instancias de producción ciudadana a los planes de fomento a la industria. Por ejemplo, se puede establecer la participación de un porcentaje de estos actores en los rodajes de las series, como colaboradores o meritorios; lo cual tendría un impacto muy positivo en el fortalecimiento de las capacidades y aprendizajes audiovisuales del sector.

c) El INCAA puede priorizar explícitamente la financiación de aquellos proyectos que busquen desarrollar procesos de alfabetización audiovisual o que incorporen material producido por instancias de producción ciudadana y por los espectadores en general.

d) Finalmente, es necesario que las instancias de producción ciudadanas puedan encontrarse para intercambiar contenidos, saberes y experiencias conjuntas, y para luchar en pos de mayores espacios de capacitación y de participación. Ya existen algunas iniciativas de estas características: la Federación Cooperativa de Productores de Contenidos Audiovisuales (FECOOP-TV), que nuclea a unas 70 organizaciones de producción audiovisual, y Trama Audiovisual, que aglomera canales cooperativos, sociedades y uniones de trabajadores (Segura, 2013, p. 11).

Acciones como las mencionadas serían complementarias al desarrollo de una industria audiovisual en crecimiento, que sin duda hay que sostener. El objetivo es lograr un cambio paulatino en la concepción de la producción audiovisual como espacio público de derecho. Esto tendría una repercusión directa en una ampliación del ejercicio de la ciudadanía comunicativa, que dejaría de estar restringida a los sectores con mayores recursos técnicos y humanos, enriqueciendo los relatos, las miradas y las experiencias de la representación audiovisual.

\section{Referencias}

Aguirre, Alix \& Fernández, Nelly (2013). Derechos humanos y tecnología comunitaria para la Inclusión social y democratización del conocimiento: proyecto de investigación. Consejo de Desarrollo Científico, Humanístico y Tecnológico de la Universidad del Zulia, Maracaibo, Venezuela. Disponible en: www.virtualeduca.info/ponencias2013/124/zuliavirtualeduca2013. docx (Consulta: 8 ago. 2015).

Argentina. Ley 26.522 (2009). Servicios de comunicación audiovisual. Disponible en http://www. infoleg.gov.ar/infolegInternet/anexos/155000-159999/158649/norma.htm (consulta: 8 ago. 2015).

Barceló, Teresa \& Sánchez, María (2010). La fuga digital: convergencia de contenidos audiovisuales interactivos en las tres pantallas: televisión, móvil e internet. En: Francés, Miquel; Gavalda, Josep; Llorca, Germán; Peris, Alvar (coords.). La calidad de los contenidos audiovisuales en la multidifusión digital (p. 181-198). Barcelona: UOC. 
Becerra, Martín (2015). De la concentración a la convergencia: políticas de medios en Argentina y América Latina. Buenos Aires: Paidós.

Bizberge, Ana; Mastrini, Guillermo \& Becerra, Martín (2011). La televisión digital terrestre en Argentina: entre la geopolítica regional y la iniciativa estatal. En: Badillo Sierra, Manuel (ed.). La transición a la televisión digital terrestre en Iberoamérica: diagnóstico y perspectiva (p. 193-222). Quito: CIESPAL.

Bressers, Hans \& Toole, Laurence (2007). Instrument selection and implementation in a networked context. En: Eliadis Pearl et al. (eds.). Designing government: from instruments to governance (p. 132-153). Montreal: McGill-Queen's University Press.

Calderón, Fernando; Hopenhayn, Martín \& Ottone, Ernesto (1996). Esa esquiva modernidad: desarrollo, ciudadanía y cultura en América Latina y el Caribe. Caracas: Nueva Sociedad.

Camacho, Carlos (2007). Cultura de la transparencia: el derecho humano a la información en el desarrollo de la ciudadanía comunicativa en Bolivia (1997-2007). La Paz: COSUDE.

Castells, Manuel (2005). La sociedad en red. Madrid: Alianza.

Escribiendocine (2010, dic. 4). Se anunciaron los ganadores de los concursos del Plan Operativo de Promoción y Contenidos Audiovisuales Digitales. Disponible en: http://www.escribiendocine. com/noticia/0002042-se-anunciaron-los-ganadores-de-los-concursos-del-plan-operativo-depromocion-y-contenidos-audiovisuales-digitales/ (consulta: 8 ago. 2015).

Guber, Rosana (2004). El salvaje metropolitano: reconstrucción del conocimiento social en el trabajo de campo. Buenos Aires: Paidós.

Halpern, Gerardo (2012). Aportes comunicacionales para un debate sobre ciudadanía y migración. Trabajo y Sociedad, 19, p. 229-242.

Hopenhayn, Martín (2000). Ciudadanía e igualdad social: ecuación pendiente. Reflexión Política. Bucaramanga, Colombia: Universidad Autónoma de Bucaramanga, 2 (3).

Instituto Nacional de Cine y Artes Audiovisuales (INCAA) (2011). Bases y condiciones para el concurso series de documentales federales. En: Plan operativo de promoción y fomento de contenidos audiovisuales digitales. $2^{\text {a }}$ ed. 2011 Disponible en: http://ant.incaa.gob.ar/castellano/ home/concursos_tvdigital/produccion_contenidos/2_documentales_federales2011.pdf (consulta: 8 ago. 2015).

Marshall, T. H. \& Bottone, T (1998). Ciudadanía y clase social. Madrid: Alianza.

Mata, María Cristina (2006). Comunicación y ciudadanía: problemas teórico-políticos de su articulación. Fronteiras: estudios mediáticos 8 (1), p. 5-15.

Segura, María Soledad (2008). Comunicación y ciudadanía en los estudios latinoamericanos de comunicación. Centro de Informaciones de la Facultad de Filosofía y Humanidades; Córdoba, Argentina. Disponible en: http://www.ffyh.unc.edu.ar/ciffyh/wp-content/uploads/2011/08/ segura-maria-soledad.pdf (consulta: 8 ago. 2015).

Segura, María Soledad (2013). El sector social en la implementación de la Ley 26.522: condiciones y estrategias. Ponencia presentada en el VI Encuentro Panamericano de Comunicación (COMPANAM), Córdoba, Argentina.

Sel, Susana (2013). La democratización comunicacional en Argentina: avances en políticas públicas y obstáculos económicos, políticos y culturales. En: Dantas, Marcos (coord.). Avances en los procesos de democratización de las comunicaciones en América Latina. (p. 139-165). Buenos Aires: Consejo Latinoamericano de Ciencias Sociales (CLACSO).

Señales (2015, ene. 21). Se conformó el Consejo Consultivo Honorario de los Medios Públicos. Disponible en: http://seniales.blogspot.com.ar/2015/01/se-conformo-el-consejo-consultivo. html (consulta: 8 ago. 2015). 
Télam (2014, sep. 22). TDA alcanzó un 87\% de cobertura nacional, dijo Osvaldo Nemirovsci. Disponible en: http://www.telam.com.ar/notas/201409/79021-osvaldo-nemirovsci-televisiondigital-abierta-cobertura-territorio-nacional-comunicacion.html (consulta: 8 ago. 2015).

\section{Contacto}

Rocío Belén Pérez Monteleone

r.perez.monteleone@gmail.com 